\title{
RELATIONSHIP BETWEEN THE JPL RADIO CELESTIAL REFERENCE FRAME AND A PRELIMINARY FK5 FRAME.
}

\author{
Jean-François Lestrade \\ Bureau des Longitudes \\ 77 Avenue Denfert-Rochereau, F-75014, Paris, France. \\ and Jet Propulsion Laboratory, California Institute of Technology, \\ 4800 Oak Grove Dr, Pasadena, California, 91109. \\ Yves Requième and Michel Rapaport \\ Observatoire de Bordeaux, F-33270, Floirac, France. \\ Robert A. Preston \\ Jet Propulsion Laboratory, California Institute of Technology, \\ 4800 Oak Grove Dr, Pasadena, California, 91109.
}

\begin{abstract}
Very Long Baseline Interferometry (VLBI) and optical positions of 8 radio stars are compared in the J2000.0 system. The mean differences in right ascension and declination found are $+0.02 " \pm 0.04 "$ and $-0.02 " \pm 0.07$ ", respectively. These differences show that the JPL radio celestial reference frame is aligned on a preliminary FK5 frame to at least this level.
\end{abstract}

\section{1- Introduction.}

The optical and radio celestial reference frames can be linked together with radio emitting stars if they can be observed by both optical and radio techniques. Such stars would be common to both reference frames and could be used as link objects. Such links should stop the possible angular rotation of the optical celestial reference frame relative to the stable extragalactic reference frame and should also unify the optical and radio coordinate systems. The Bordeaux Automatic Meridian Circle and the Very Long Baseline Interferometry (VLBI) technique were used to derive the positions of optically bright radio stars in the FK4 and in the Jet Propulsion Laboratory (JPL) radio celestial reference frame, respectively. These positions are compared here and the relationship between a preliminary FK5 frame and the JPL radio celestial reference frame is discussed.

\section{2 - Optical and VLBI positions of 8 radio stars.}

The Bordeaux Automatic Meridian Circle (Requième 1973) measures the positions of stars relative to a large number of FK4 fundamental stars (eventually FK5). Briefly, the program stars of a night are observed with about 40 fundamental stars spread over a very large area of the sky (about 4 hours in right ascension and from -15 to 75 degrees in 
declination). These FK4 stars are used to derive the instrument parameters for each night. Hence, the positions of the program stars are measured directly in the FK4 reference frame. This strategy of observations averages out the regional distortions of the FK4 which are as large as a few 0.1" and the final accuracy of the positions of the Bordeaux Automatic Meridian Circle is expected to be about 0.05" after adding the data of about ten nights. This instrument routinely measures the positions of about 250 program stars per night for the HIPPARCOS project. Optically bright radio stars observed by the VLBI technique and the Very Large Array (VLA) are part of this program since they will be used to link the HIPPARCOS stellar frame to the stable VLBI extragalactic reference frame.

Very Long Baseline arrays at centimeter wavelengths have been used to observe successfully 8 compact radio stars during moderate or strong outbursts when their flux densities were between 10 and 400 milliJansky. These observations have been conducted since late 1982 with the sensitive Mark III VLBI data acquisition system (Rogers et al 1983) and with the most sensitive radiotelescopes. The raw data were correlated at Haystack Observatory. The observing strategy included the observations of about 20 radio extragalactic sources with milliarcsecond positions in the JPL radio celestial reference frame in addition to the program radio stars. These extragalactic sources were used as position calibrators to determine the clock and the tropospheric parameters. These VLBI observations were conducted at a single frequency (mostly at $5 \mathrm{GHz}$ and $8.4 \mathrm{GHz}$ ) and a model was used to correct for the ionosphere. The locations of the radiotelescopes were adopted from VLBI determinations of the Crustal Dynamics (CDP) and JPL programs and the Earth orientation parameters were adopted from the IRIS monitoring program. The consistency between the adopted celestial reference frame (JPL), Earth orientation parameters (IRIS) and terrestrial reference frame (CDP and JPL) might be insufficient for comparison at the milliarcsecond level (see Arias, Lestrade and Feissel in these proceedings) but it is thought to be sufficient at the 0.01 " precision level. VLBI positions of the 8 compact radio stars (Lestrade et al 1986) were determined with the astrometric VLBI package MASTERFIT (Fanselow and Sovers 1985) in the J2000.0 system with typical precisions of 0.01 " and better in two cases. These stars were also observed at Bordeaux and their B1950 positions derived by Requième et al (1986a).

\section{3 - Comparison of the optical and VLBI positions of 8 radio stars.}

The optical and VLBI positions were measured at different dates and are reduced relative to the mean equators and equinoxes of epoch B1950 and J2000, respectively. Therefore the B1950 optical positions were first shifted to the dates of the VLBI observations with the B1950 proper motions published by Johnston et al (1985) and de Vegt et al (1985). The differences between observation dates are small (about 3 years) and these proper motions are accurate enough to produce reliable positions at the shifted epochs. Then, these B1950 optical positions were transformed to the J2000 mean equator and equinox in using the transformation matrix devised by Aoki et al (1983). This procedure includes the equinox correction of Fricke (1982) and yields J2000 positions which are in the FK5 system. Finally, we have compared these FK5 optical positions to the J2000 VLBI positions of the 8 radio stars and the differences in right ascension and declination are in table 1. The last two lines of this table report the mean and standard deviation of the differences for each coordinate. 


\begin{tabular}{|l|r|r|}
\hline \multicolumn{1}{|c|}{ Stars } & $\begin{array}{r}\cos \delta \Delta \alpha \\
\left(0.01{ }^{\prime}\right)\end{array}$ & $\begin{array}{c}\Delta \delta \\
(0.01 ")\end{array}$ \\
\hline LSI 61303 & $(-16)$ & $(+1)$ \\
Algol & +1 & -1 \\
UX Arietis & 0 & +1 \\
HR 1099 & +4 & -12 \\
HR 5110 & 0 & +7 \\
$\sigma$ CrB & -3 & -3 \\
Cyg X1 & +6 & +3 \\
SZ Psc & +9 & -14 \\
\hline Mean $(\Delta \alpha, \Delta \delta)$ & +2 & -2 \\
Standard deviation & 4 & 7 \\
\hline
\end{tabular}

Table 1: Differences between optical and VLBI coordinates of 8 radio stars relative to the J2000 mean equator and equinox. The differences are in the sense optical minus VLBI. The position of LSI 61303 is preliminary and is not included in the mean and standard deviation.

\section{4 - Discussion.}

The transformation of Aoki et al (1983) includes the change in the conventional value of the general precession from the B1950 system to the new J2000 system. It includes also the rotation of the FK4 relative to the FK5 calculated by Fricke (1982) as a single equinox correction since he found that the mean equators of the two catalogues are coincident at any common epoch. This equinox correction and the new convention system define the FK5 reference frame as recommended by the IAU. Presently however, no direct reduction can be made in this new frame since its materialization by a catalogue is not available yet. So the optical positions from Bordeaux which are first reduced in the FK4 and then transformed by Aoki et al (1983) are defining a preliminary FK5 frame.

The mean differences that we have found are 0.02 " \pm 0.04 " in $\alpha$ and $-0.02 " \pm 0.07$ " in $\delta$ in using the standard deviations as realistic uncertainties here. It is interesting to note first that these uncertainties are expected if the VLBI precision ( 0.01 ") and the optical precision ( $0.05 ")$ quoted above are combined. These differences show that the JPL radio celestial reference frame is aligned on our preliminary FK5 frame to at least this accuracy.

Such a good alignment in right ascension is fortuitous since the origin of the VLBI right ascension is chosen by adopting the right ascension of the source 3C273B derived by Hazard et al (1971) with an uncertainty of 0.2 " from optical observations and radio occultations of this source by the Moon. Requième et al (1986b) have recently measured a new optical position of $3 \mathrm{C} 273 \mathrm{~B}$ with the Bordeaux Automatic Meridian Circle and found that its right ascension agrees with the value measured by Hazard et al (1971) at least at the 0.05 " level.

Newhall, Preston and Esposito (1984) have determined offsets of $0.00 " \pm 0.04$ " in $\alpha$ and $0.01 " \pm 0.05 "$ in $\delta$ between the dynamical reference frame associated with the ephemerides of the interior planets and the JPL radio celestial reference frame. These small offsets are also consistent with our result since the FK5 system is defined as close as possible to the dynamical reference frame at epoch J2000 as recommended by the IAU (Fricke 1982). 
Finally, the differences in $\alpha$ and $\delta$ of table 1 are consistent with a previous comparison made between the same VLBI positions and earlier Bordeaux optical positions (Lestrade et al 1985).

\section{Acknowledgments:}

The research described in this paper was carried out, in part, by the Jet Propulsion Laboratory, California Institute of Technology, under contract with the National Aeronautics and Space Administration and jointly with the Groupe de Recherche en Géodésie Spatiale trough agreeements with the National Aeronautics and Space Administration.

\section{References:}

Aoki, S., Soma, M., Kinoshita, H., Inoue, K., 1983,Astron. Astroph., 128, 263.

de Vegt, C., Florkowsky, D.R., Johnston, K.J., Wade, C.M., 1985, A.J.,90, 2387.

Fanselow, J.L., Sovers, O.J., 1985, Jet Propulsion Laboratory Publication 89-99, Rev. 1. Fricke, W., 1982, Astron. Astroph., 107, L13.

Hazard, C., Sutton, J. Argue, A.N., Kenworthy, C.M., Morrison, L.V., Murray, C.A., 1971, Nature, 233, 89.

Johnston, K.J., Wade, C.M., Florkowsky, D.R., de Vegt, C., 1985, A.J., 90, 1343.

Lestrade, J.-F., Preston, R.A., Requième, Y., Rapaport, M., Mutel, R.L., 1985, Proceedings of a colloquium on "the European Astrometry Satellite HIPPARCOS Scientific Aspects of the Input Catalogue Preparation", Aussois, France, 3-7 June 1985, European Space Agency SP-234, p. 251.

Lestrade, J.-F., Preston, R.A., Niell, A.E., Mutel, R.L., 1986, in preparation for A.J.

Newhall, XX, Preston, R.A., Esposito, P.B., 1984, Proceedings of the IAU Symposium No 109: Astrometric Techniques, edtited by H. K. Einchhorn and Leacock, R.J., Reidel publisher, 779.

Requième, Y., 1973, Astron. Astrophys., 23, 453.

Requième, Y., et al, 1986a, in preparation.

Requième, Y., et al, 1986b, in preparation.

Rogers, A.E.E. et al, 1983, Science, 219, 51.

\section{DISCUSSION}

Hughes: I have a question and a comment. The question: are the same 40 FK4 stars observed every night? The comment: I would not equate the FK4 on J2000 by using the Aoki procedure with the FK5 since the individual corrections are not included and, especially in your case, systematics such as $\Delta \alpha_{\alpha}$ could cause significant errors.

Reply by Lestrade: Forty FK4 stars are observed every clear night by the Bordeaux Automatic Meridian Circle. Not the same FK4 stars are scheduled each night. The Bordeaux optical positions are not directly reduced in the FK5 for this system is not yet materialised by a catalogue. Nevertheless, the directions of axes of the FK5 are known, given by Fricke in 1982: the FK4 and FK5 equators are coincident and the equinox correction is provided. The preliminary FK5 referred to in this paper is incomplete since we do not know yet the zonal corrections to apply for the FK4 stars. This was well understood in chosing the terminology "preliminary FK5."

T. Clark: Just a comment - the uncertainty in the right ascension of 3C273B from Hazard et. al. was dominated by the corrections due to the lunar limb, and their $0^{\prime \prime} .2$ as quoted by the author was probably conservative.

Reply by Lestrade: Recent results seem to indicate that their uncertainty was indeed conservative. 